\title{
Gene Signature for Sorafenib Susceptibility in Hepatocellular Carcinoma: Different Approach with a Predictive Biomarker
}

\author{
Chang Min Kim ${ }^{a}$ Shin Hwang ${ }^{b}$ Bhumsuk Keam ${ }^{c}$ Yun Suk Yu ${ }^{a}$ Ji Hoon Kim ${ }^{d}$ \\ Dong-Sik Kim ${ }^{e}$ Si Hyun Bae ${ }^{f}$ Gun-Do Kima, Jong Kyu Lee ${ }^{a}$ Yong Bae Seo ${ }^{a}$ \\ Soon Woo Nam ${ }^{\text {h }}$ Koo Jeong Kang ${ }^{i}$ Luigi Buonaguroj Jin Young Park ${ }^{a}$ \\ Yun Soo Kimk ${ }^{k}$ Hee Jung Wangl \\ ${ }^{a}$ CbsBioscience Inc., Daejeon, South Korea; ${ }^{b}$ Division of Hepatobiliary Surgery and Liver \\ Transplantation, Department of Surgery, Asan Medical Center, University of Ulsan College \\ of Medicine, Seoul, South Korea; ${ }^{c}$ Department of Internal Medicine, Seoul National \\ University Hospital, Seoul National University College of Medicine, Seoul, South Korea; \\ ${ }^{\mathrm{d}}$ Division of Gastroenterology and Hepatology, Department of Internal Medicine, Korea

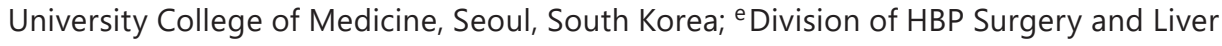 \\ Transplantation, Department of Surgery, Korea University College of Medicine, Seoul, \\ South Korea; ${ }^{f}$ Department of Internal Medicine, Eunpyeong St. Mary's Hospital, College \\ of Medicine, The Catholic University of Korea, Seoul, South Korea; 9 Department of \\ Microbiology, College of Natural Sciences, Pukyong National University, Busan, South \\ Korea; ${ }^{\text {h}}$ Department of Internal Medicine, Incheon St. Mary's Hospital, The Catholic \\ University of Korea, Incheon, South Korea; 'Division of Hepatobiliary and Pancreatic \\ Surgery, Department of Surgery, Keimyung University Dong-San Medical Center, Daegu, \\ South Korea; 'Laboratory of Cancer Immunoregulation, Instituto Nazionale per lo \\ Studio e la Cura dei Tumori "Fondazione G. Pascale" - IRCCS, Naples, Italy; ${ }^{\text {kDivision of }}$ \\ Gastroenterology and Hepatology, Department of Internal Medicine, Gil Medical Center, \\ Gachon University College of Medicine, Incheon, South Korea; 'Department of Surgery, \\ Ajou University School of Medicine, Suwon, South Korea
}

\section{Keywords}

Sorafenib · Biomarker · Gene signature · Hepatocellular carcinoma

\begin{abstract}
Background/Aim: Uniform treatment of hepatocellular carcinoma (HCC) with molecular targeted drugs (e.g., sorafenib) results in a poor overall tumor response when tumor subtyping is absent. Patient stratification based on actionable gene expression is a method that can potentially improve the effectiveness of these drugs. Here we aimed to identify the clinical applica-
\end{abstract}

Chang Min Kim and Shin Hwang, as well as Hee Jung Wang and Yun Soo Kim, contributed equally to this work.

Yun Soo Kim, MD, PD

Department of Internal Medicine, Gil Medical Center

Gachon University College of Medicine

Namdong-daero, Namdong-gu, Incheon 21565 (South Korea)

kimys@gilhospital.com
Hee Jung Wang, MD, PhD

Department of Surgery, Ajou University Hospital

World Cup-ro, Yeongtong-gu

Suwon 16499 (South Korea)

wanghj@ajou.ac.kr 
tion of actionable genes in predicting response to sorafenib. Methods: Through quantitative real-time reverse transcription $P C R$, we analyzed the expression levels of seven actionable genes (VEGFR2, PDGFRB, C-KIT, C-RAF, EGFR, mTOR, and FGFR1) in tumors versus noncancerous tissues from $220 \mathrm{HCC}$ patients treated with sorafenib. Our analysis found that 9 responders did not have unique clinical features compared to nonresponders. A receiver operating characteristic curve evaluated the predictive performance of the treatment benefit score (TBS) calculated from the actionable genes. Results: The responders had significantly higher TBS values than the nonresponders. With an area under the curve of 0.779, a TBS combining mTOR with VEGFR2, c-KIT, and c-RAF was the most significant predictor of response to sorafenib. When used alone, sorafenib had a $0.7-3 \%$ response rate among HCC patients, but when stratifying the patients with actionable genes, the tumor response rate rose to $15.6 \%$. Furthermore, actionable gene expression is significantly correlated with tumor response. Conclusions: Our findings on patient stratification based on actionable molecular subtyping potentially provide a therapeutic strategy for improving sorafenib's effectiveness in treating HCC.

(C) 2020 The Author(s)

Published by S. Karger AG, Basel

\section{Introduction}

Hepatocellular carcinoma (HCC) is a lethal malignancy that is notoriously resistant to chemotherapy [1], leading to a search for alternative treatment strategies. Among these, molecular targeted drugs are designed to antagonize "oncogenic-actionable genes" that are specifically altered in tumors [2]. Actionable molecules have been successfully applied to patient stratification in various cancers [3]. Lung cancer can be classified into 18 subtypes based on specificgenetic aberrations (e.g., overexpression and mutations) [4], while melanoma has 11 subtypes associated with targeted treatment [5]. Unfortunately, the highly heterogeneous HCC lacks molecular predictors of treatment response. Although many molecular targeted agents have been tested in HCC, only sorafenib and lenvatinib have been approved as first-line agents for advanced stages of this cancer [6-8]. The lack of a second-line option after lenvatinib [9], however, means that sorafenib is the recommended standard treatment for advanced HCC [9], despite its poor therapeutic response $(0.7-3 \%)[6,7]$. New therapeutic strategies are necessary to improve sorafenib's efficacy in treating HCC.

Currently, there is no clinically applicable biomarker for predicting HCC response to sorafenib [10], although VEGFA [11] and FGF3/4 [12] are more frequently amplified in sorafenib responders. Previously, we investigated the mRNA expression of five actionable genes (VEGFR2, PDGFRB, EGFR, $m$ TOR, and FGFR1) in HCC tumors and surrounding noncancerous tissues, and then used the results to stratify HCC patients. Additionally, we found evidence suggesting that $c-R A F$ and $P D G F R B$ expression could predict sorafenib susceptibility [13].

In this study, we aimed to confirm whether actionable gene expression can predict response to sorafenib in HCC tissues, and if so, identify those with the best diagnostic performance. We compared the mRNA expression of seven actionable genes in responder and nonresponder tumor tissues from 220 HCC patients treated with sorafenib. We then calculated and compared treatment benefit scores (TBS) from relative mRNA levels per gene.

\section{Subjects and Methods}

Patients and Tissue Samples

Patients with histologically confirmed HCC were enrolled if they met the following criteria: (1) age $\geq 20$ years; (2) diagnosed with unresectable advanced HCC; (3) Eastern Cooperative Oncology Group performance status $\leq 2$; (4) Child-Pugh class A; and (5) receiving sorafenib as a palliative first-line systemic 


\section{Liver Cancer}

\begin{tabular}{|c|c|}
\hline Liver Cancer 2020;9:182-1 & \\
\hline DOI: $10.1159 / 000504548$ & $\begin{array}{l}\text { (c) } 2020 \text { The Author(s). Published by S. Karger AG, Basel } \\
\text { www.karger.com/lic }\end{array}$ \\
\hline
\end{tabular}

Kim et al.: Gene Signature as Predictive Biomarker of Sorafenib Susceptibility

treatment. Individuals were excluded if they required combination therapy (including chemotherapy, radiotherapy, hepatic arterial chemoembolization, and radiofrequency ablation) or possessed severe, uncontrolled medical conditions. In total, 390 patients were enrolled from 7 medical institutions; all provided written informed consent. Sorafenib treatment occurred during 2014-2018. Inoperable patients were subjected to ultrasound-guided needle biopsy before sorafenib treatment. For patients experiencing recurrence within 3 months after surgical resection with curative intent, needle biopsy was omitted. Instead, tumor tissues frozen at the time of resection were used for analysis.

Complete clinical information was available for all cases. Radiologic analyses (computed tomography [CT] and magnetic resonance imaging [MRI]) evaluated tumor response to sorafenib, following the modified Response Evaluation Criteria in Solid Tumors for HCC [14]. Immediately after needle biopsy, HCC tissue samples were snap-frozen in liquid nitrogen and stored at $-80^{\circ} \mathrm{C}$. Patient staging information was obtained from CT or MR images, and conventional TNM (Tumor, Node, and Metastasis) classification (American Joint Committee on Cancer, 7th edition), along with BCLC (Barcelona Clinic Liver Cancer) staging, was used.

\section{Measurement of Clinical Outcomes}

The primary endpoint was tumor response to sorafenib, assessed 3 and 6 months after drug administration.

\section{RNA Extraction and CDNA Synthesis}

Published methods were used for RNA extraction and cDNA synthesis [13]. Total RNA was extracted from both tumor and surrounding noncancerous frozen tissues using the RNeasy Mini Kit (Qiagen, Hilden, Germany) with DNase I treatment (Qiagen). Total RNA integrity was verified using a Bioanalyzer 2100 (Agilent Technologies, Santa Clara, CA, USA). Next, $4 \mu \mathrm{g}$ of RNA was incubated with $2 \mu \mathrm{L}$ of $10 \mu \mathrm{m}$ oligo(dT)18 primer (GenoTech, Daejeon, South Korea) at $70^{\circ} \mathrm{C}$ for $7 \mathrm{~min}$, before being cooled on ice for $5 \mathrm{~min}$. Reverse transcriptase enzyme mix was added to the annealed total RNA sample, and the reaction was incubated for $90 \mathrm{~min}$ at $42^{\circ} \mathrm{C}$. Reverse transcriptase was then heat-inactivated at $80^{\circ} \mathrm{C}$ for $10 \mathrm{~min}$. Diethylpyrocarbonatetreated water was added to bring the final volume of the cDNA samples to $400 \mu \mathrm{L}$.

\section{Quantitative Real-Time PCR}

Quantitative real-time PCR was performed as described previously [13], using an ABI PRISM 7900HT instrument (Applied Biosystems, Foster City, CA, USA). The total reaction volume was $10 \mu \mathrm{L}$. The thermocycling conditions were as follows: $95^{\circ} \mathrm{C}$ for $10 \mathrm{~min}$, followed by 45 cycles of $95^{\circ} \mathrm{C}$ for $15 \mathrm{~s}$ and $60^{\circ} \mathrm{C}$ for $1 \mathrm{~min}$. The primer and probe sequences were designed in Primer Express 3.0 (Applied Biosystems); all probes were labeled with TAMRA at the $3^{\prime}$ end and FAM at the $5^{\prime}$ end. The target genes were $m T O R, V E G F R 2$, PDGFRB, FGFR1, c-KIT, EGFR, and c-RAF. The internal control was the average expression of five reference genes $\left(\beta_{2^{-}}\right.$ microglobulin $[B 2 M]$, glyceraldehyde 3-phosphate dehydrogenase $[G A P D H]$, hydroxymethylbilane synthase [HMBS], hypoxanthine phosphoribosyltransferase 1 [HPRT1], and succinate dehydrogenase complex, subunit A, flavoprotein variant [SDHA]). The $2^{-\Delta C t}$ method was used to determine target gene expression levels.

\section{Treatment Benefit Score}

The TBS is the summation of all $\log _{2}$-transformed target gene expression multiplied by its corresponding regression coefficient, as follows: TBS $=(0.118910 \times m T O R)+(0.138561 \times V E G F R 2)+(0.258877 \times c-K I T)+$ $(0.147012 \times c-R A F)$. The regression coefficients of each gene were calculated by logistic regression analysis with the R package (version 3.3.3; R Development Core Team; https://www.r-project.org/).

\section{Statistical Analysis}

Receiver operating characteristic curve analysis was used to determine the accuracy of the threshold values separating tumor responders and nonresponders using the TBS. Relationships between tumor response and clinicopathologic variables or gene expression were evaluated using either $\chi^{2}$ tests or Fisher's exact tests. Gene expression data were $\log _{2}$ transformed and tested for normality with the Shapiro-Wilk test. As the data did not meet the normality assumptions, significant differences between responders and nonresponders were evaluated using the Mann-Whitney U test. Every tested gene from the 220 patients was analyzed using logistic regression to understand the relationships between response to sorafenib treatment, TBS classification, and clinicopathologic variables. Significance was set at $p<0.05$ (two-tailed). All statistics were performed in $\mathrm{R}$ version 3.3.3. 


\section{Liver Cancer}

Fig. 1. Flowchart of treatment enrollment and follow-up. HCC, hepatocellular carcinoma; CR, complete response; $\mathrm{PR}$, partial response; SD, stable disease; $\mathrm{PD}$, progressive disease.

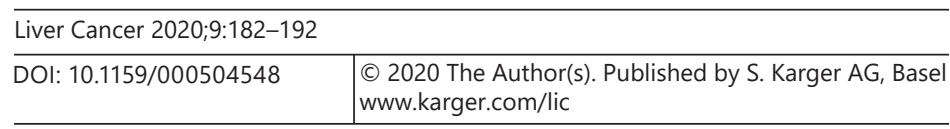

Kim et al.: Gene Signature as Predictive Biomarker of Sorafenib Susceptibility

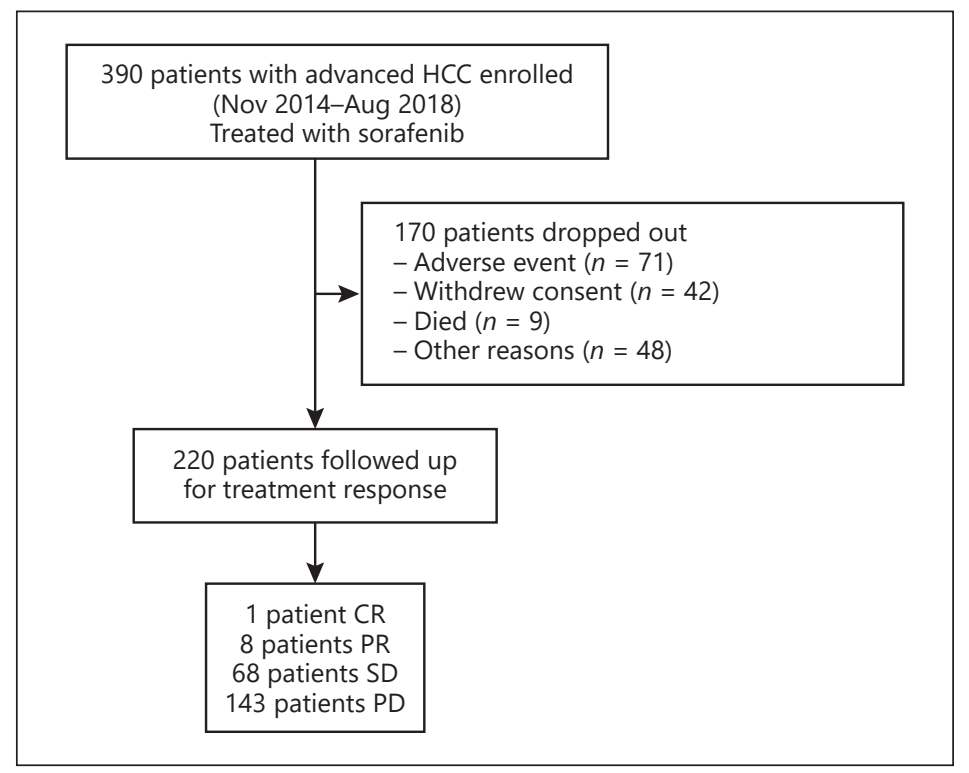

\section{Results}

\section{Clinicopathologic Characteristics of the Sorafenib Responders}

Of the 390 sorafenib-treated patients, 220 were retained for follow-up treatments. The remainder dropped out due to adverse events $(n=71)$, withdrawal of consent $(n=42)$, death $(n=9)$, or other reasons ( $n=48)$ (Fig. 1$)$.

The results of the CT and MRI scans revealed 1 patient with a complete response, 8 with a partial response, 68 with stable disease, and the remaining 143 with progressive disease. The overall tumor response rate was $4.1 \%$. Representative tumors presented a dramatic disappearance of nodules in the lung and liver after sorafenib treatment (Fig. 2). An analysis of clinicopathologic features associated with response to sorafenib revealed variable $\alpha$-fetoprotein levels among responders, ranging from 1 to $14,046 \mathrm{ng} / \mathrm{mL}$ (Table 1). A comparison between responders and nonresponders did not reveal any clinical features unique to responders (Table 2).

\section{Comparison of Actionable Gene Expression between Responders and Nonresponders}

In a comparison across responders and nonresponders to sorafenib, we found that five genes (mTOR: $p=0.309 ;$ PDGFRB: $p=0.119 ; c-K I T: p=0.014 ; c-R A F: p=0.002$; and EGFR: $p=$ 0.137 ) had a higher mean expression in the responders than in the nonresponders (Fig. 3a, $c-f)$. However, the mean VEGFR2 $(p=0.224)$ and FGFR1 $(p=0.475)$ expression was lower in the responders than in the nonresponders (Fig. 3b, g).

\section{Comparing TBS between Responders and Nonresponders}

We found that combinations of $m T O R, V E G F R 2, c-K I T$, and $c-R A F$ were most effective in discriminating responders from nonresponders. Compared with the TBS of a single gene ( $m T O R, V E G F R 2, c-K I T$, or $c-R A F$ ), the TBS of $m T O R / V E G F R 2$ and $c-K I T / c-R A F$ combinations differed significantly between the responders and the nonresponders (Fig. 4a). The mean TBS of $m$ TOR/VEGFR2 and $c-K I T / c-R A F$ was -10.110464 in the nonresponders and -7.805714 in the responders, respectively $(p<0.001)$. 
Kim et al.: Gene Signature as Predictive Biomarker of Sorafenib Susceptibility

Table 1. Clinicopathologic features of sorafenib responders

\begin{tabular}{lllllllll}
\hline $\begin{array}{l}\text { Patient } \\
\text { No. }\end{array}$ & $\begin{array}{l}\text { Age, } \\
\text { years }\end{array}$ & Sex & $\begin{array}{l}\text { Viral } \\
\text { status }\end{array}$ & $\begin{array}{l}\text { TNM stage } \\
\text { (AJCC } \\
\text { 7th ed.) }\end{array}$ & $\begin{array}{l}\text { BCLC } \\
\text { stage }\end{array}$ & $\begin{array}{l}\text { AFP, } \\
\text { ng/mL }\end{array}$ & Metastasis & $\begin{array}{l}\text { Tumor } \\
\text { response }\end{array}$ \\
\hline 1 & 60 & F & HBV & IV & C & 1.6 & X & PR \\
2 & 46 & F & HBV & IV & C & 489.7 & O & PR \\
3 & 52 & F & HBV & IV & B & 6,519 & O & PR \\
4 & 52 & M & HBV & III & C & $14,046.0$ & X & PR \\
5 & 56 & M & HBV & IV & B & 10.1 & O & PR \\
6 & 38 & M & HBV & III & C & 7.6 & X & PR \\
7 & 43 & M & HBV & IV & C & 617.0 & O & PR \\
8 & 56 & M & HBV & IV & C & 3 & X & CR \\
9 & 62 & M & HBV & IV & C & 3.9 & X & PR \\
\hline
\end{tabular}

AJCC, American Joint Committee on Cancer; BCLC, Barcelona Clinic Liver Cancer; AFP, $\alpha$-fetoprotein; HBV, hepatitis $\mathrm{B}$ virus; $\mathrm{CR}$, complete response; $\mathrm{PR}$, partial response.

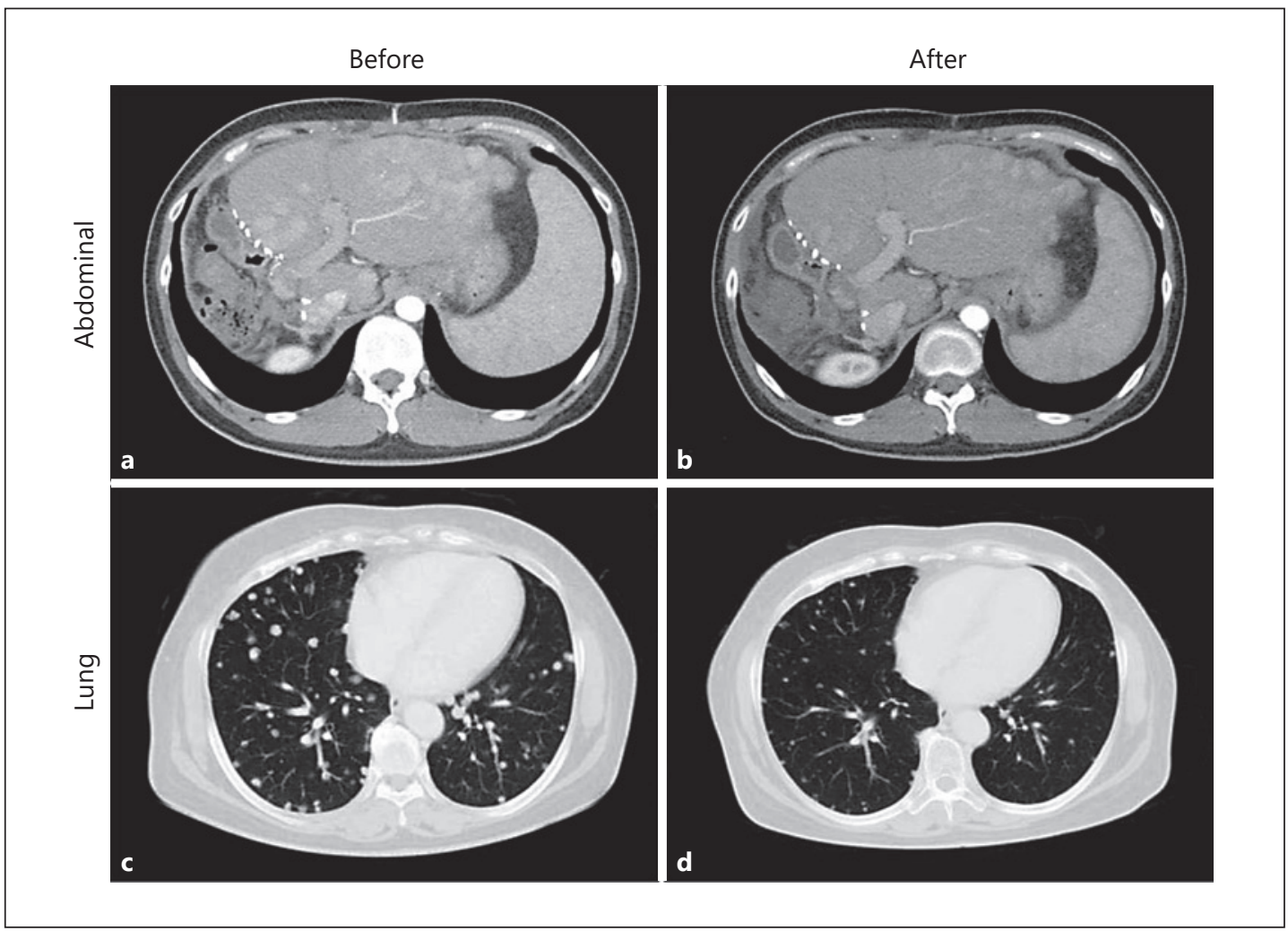

Fig. 2. Abdominal (a, b) and lung (c, d) images from representative responders. CT images taken before (a, c) and after (b, d) sorafenib treatment, showing changes in multiple nodular lesions spread in the organs.

Performance of Gene Classifiers in Predicting Tumor Response to Sorafenib

Multiple actionable genes were differentially expressed in tumor tissues between responders and nonresponders. We performed a receiver operating characteristic curve analysis of genes in combination; the number of genes combined was from 1 to 7 . Among all the gene combinations, 9 of the classifiers revealed over $70 \%$ in sensitivity and specificity. 


\section{Liver \\ Cancer}

Table 2. Baseline characteristics and comparison of clinical factors between responders and nonresponders

\begin{tabular}{|c|c|}
\hline \multicolumn{2}{|l|}{ Liver Cancer 2020;9:182-192 } \\
\hline DOI: $10.1159 / 000504548$ & $\begin{array}{l}\text { (c) } 2020 \text { The Author(s). Published by S. Karger AG, Basel } \\
\text { www.karger.com/lic }\end{array}$ \\
\hline
\end{tabular}

Kim et al.: Gene Signature as Predictive Biomarker of Sorafenib Susceptibility

\begin{tabular}{|c|c|c|c|}
\hline $\begin{array}{l}\text { Clinicopathologic } \\
\text { parameters }\end{array}$ & $\begin{array}{l}\text { Responders } \\
(n=9)\end{array}$ & $\begin{array}{l}\text { Nonresponders } \\
(n=211)\end{array}$ & $p$ value $^{1}$ \\
\hline \multicolumn{2}{|l|}{ Age } & $(-1)$ & 0.7384 \\
\hline$<55$ years & 3 & 88 & \\
\hline$\geq 55$ years & 6 & 122 & \\
\hline \multicolumn{2}{|l|}{ Gender } & $(-1)$ & 0.4148 \\
\hline Male & 6 & 165 & \\
\hline Female & 3 & 45 & \\
\hline \multicolumn{2}{|l|}{ HBV } & $(-1)$ & 0.3627 \\
\hline Absent & 0 & 37 & \\
\hline Present & 9 & 173 & \\
\hline \multicolumn{2}{|l|}{$\mathrm{HCV}$} & $(-2)$ & 1 \\
\hline Absent & 9 & 200 & \\
\hline Present & 0 & 9 & \\
\hline \multicolumn{2}{|c|}{ Tumor TNM stage (AJCC 7th ed.) } & $(-6)$ & 0.7607 \\
\hline I & 0 & 0 & \\
\hline II & 0 & 10 & \\
\hline III & 2 & 29 & \\
\hline IV & 7 & 166 & \\
\hline \multicolumn{2}{|l|}{ BCLC stage } & $(-2)$ & 0.4223 \\
\hline $\mathrm{A}$ & 0 & 3 & \\
\hline $\mathrm{B}$ & 2 & 23 & \\
\hline $\mathrm{C}$ & 7 & 181 & \\
\hline D & 0 & 2 & \\
\hline \multicolumn{2}{|l|}{ AFP } & $(-14)$ & 0.7363 \\
\hline$<100 \mathrm{ng} / \mathrm{mL}$ & 5 & 91 & \\
\hline$\geq 100 \mathrm{ng} / \mathrm{mL}$ & 4 & 106 & \\
\hline \multicolumn{4}{|l|}{ Tumor response } \\
\hline Complete response & 1 & 0 & \\
\hline Partial response & 8 & 0 & \\
\hline Stable disease & 0 & 68 & \\
\hline Progressive disease & 0 & 143 & \\
\hline
\end{tabular}

HBV, hepatitis B virus; HCV, hepatitis C virus; TNM, Tumor, Node, and Metastasis classification; AJCC, American Joint Committee on Cancer; BCLC, Barcelona Clinic Liver Cancer; AFP, $\alpha$-fetoprotein. ${ }^{1} p$ values were calculated using Fisher's exact test.

The performance of the best classifier (combinations of $m T O R, V E G F R 2, c-K I T$, and $c-R A F$ ) was at the threshold value at -8.6237 ; the prediction sensitivity in separating responders from nonresponders was $77.8 \%$ (95\% CI: 40.0-97.2), while the specificity was $82.0 \%$ (95\% CI: 76.1-86.9) (Fig. 4c). Additionally, the area under the curve was 0.779 (95\% CI: 0.724-0.833), which was statistically significant $(p<0.001$; Table 3$)$.

\section{TBS-Based Classifiers and Clinicopathologic Characteristics Both Predict Response to}

\section{Sorafenib Treatment}

Logistic regression demonstrated that TBS-based classifiers stemming from $m T O R$, VEGFR2, $c-K I T$, and $c-R A F$ combinations significantly predicted tumor response to sorafenib treatment $(p<0.001$; Table 4). Logistic regression also showed that none of the measured clinicopathologic variables (age: $p=0.611$; sex: $p=0.404$; hepatitis B virus: $p=0.993$; hepatitis C virus: $p=0.994$; TNM stage: $p=0.812$; BCLC stage: $p=0.399$; and $\alpha$-fetoprotein levels: $p=0.373$ ) significantly predicted response to sorafenib (Table 4 ). 


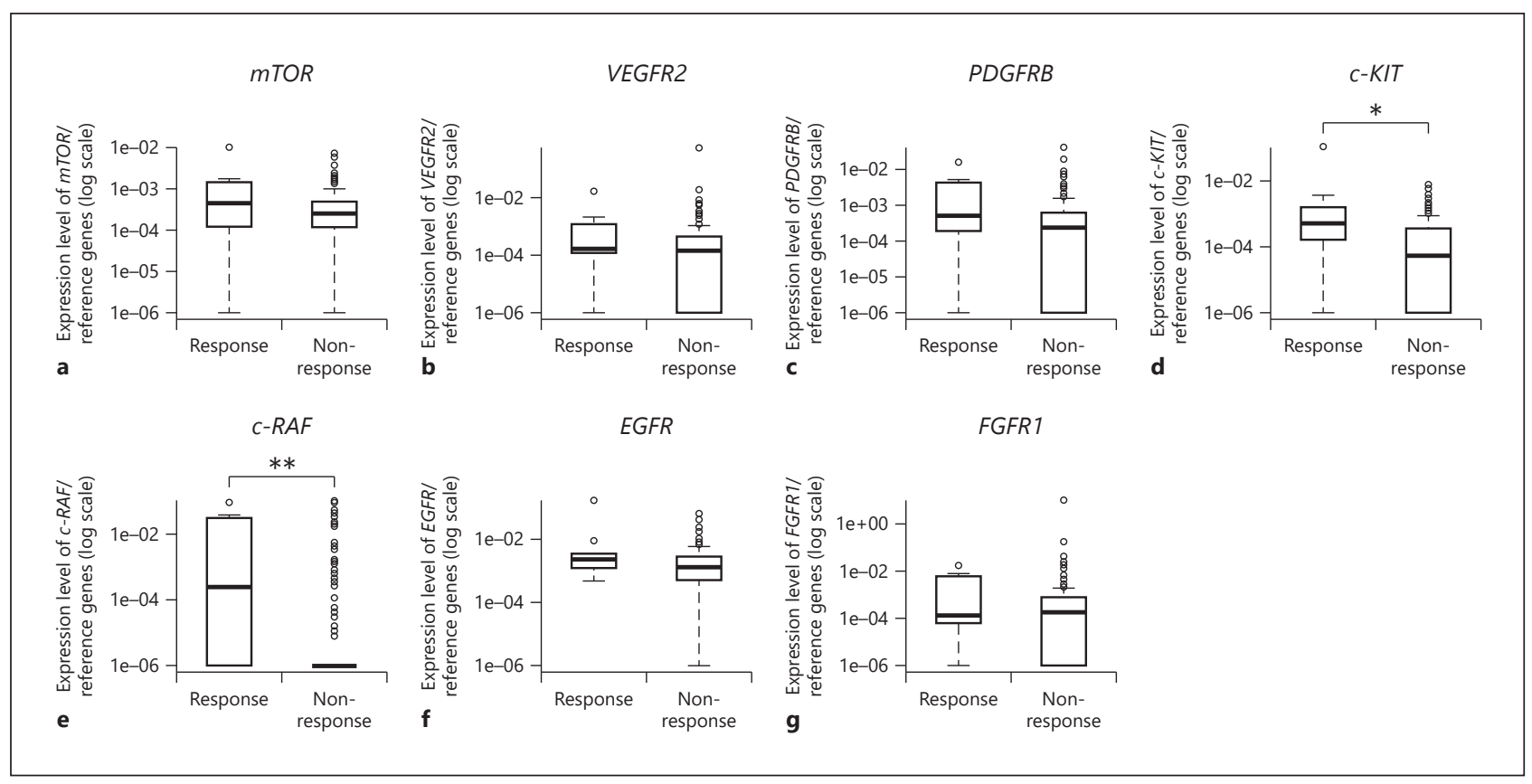

Fig. 3. Actionable gene expression in responders versus nonresponders. Relative expression of seven actionable genes in 9 responders and 211 nonresponders. ${ }^{*} p<0.05,{ }^{* *} p<0.01$. a mTOR. b VEGFR2. c PDGFRB. d $c-K I T$. e $c-R A F$. f EGFR. g FGFR1.

Table 3. Classifiers of tumor response to sorafenib

\begin{tabular}{|c|c|c|c|c|c|c|c|c|}
\hline Rank & Gene combination & $\begin{array}{l}\text { Combi- } \\
\text { nation }\end{array}$ & $\begin{array}{l}\text { Sensitivity, } \\
\%\end{array}$ & $\begin{array}{l}\text { Specificity, } \\
\%\end{array}$ & AUC & Accuracy & PPV & NPV \\
\hline 1 & $m T O R \_V E G F R 2 \_c-K I T \_c-R A F$ & 4 & 77.78 & 81.99 & 0.779 & 81.82 & 15.56 & 98.86 \\
\hline 2 & $m T O R \_c-K I T_{-} c-R A F$ & 3 & 77.78 & 81.52 & 0.778 & 81.36 & 15.22 & 98.85 \\
\hline 3 & mTOR_VEGFR2_c-KIT_c-RAF_FGFR1 & 5 & 77.78 & 81.52 & 0.770 & 81.36 & 15.22 & 98.85 \\
\hline 4 & VEGFR2_c-KIT_c-RAF & 3 & 77.78 & 80.57 & 0.781 & 80.45 & 14.58 & 98.84 \\
\hline 5 & mTOR_c-KIT_c-RAF_FGFR1 & 4 & 77.78 & 80.09 & 0.764 & 80.00 & 14.29 & 98.83 \\
\hline 6 & $c-K I T_{-} c-R A F$ & 2 & 77.78 & 77.73 & 0.791 & 77.73 & 12.96 & 98.80 \\
\hline 7 & $c-K I T_{-} c-R A F_{-} F G F R 1$ & 3 & 77.78 & 77.73 & 0.769 & 77.73 & 12.96 & 98.80 \\
\hline 8 & VEGFR2_c-KIT_c-RAF_FGFR1 & 4 & 77.78 & 77.25 & 0.765 & 77.27 & 12.73 & 98.79 \\
\hline \multirow[t]{9}{*}{9} & mTOR_VEGFR2_c-KIT_c-RAF_EGFR & 5 & 77.78 & 73.46 & 0.769 & 73.46 & 11.11 & 98.73 \\
\hline & mTOR & 1 & 44.44 & 87.68 & 0.600 & 85.91 & 13.33 & 97.37 \\
\hline & VEGFR2 & 1 & 88.89 & 36.02 & 0.618 & 38.18 & 5.59 & 98.70 \\
\hline & $c-K I T$ & 1 & 88.89 & 56.87 & 0.731 & 58.18 & 8.08 & 99.17 \\
\hline & $c-R A F$ & 1 & 55.56 & 86.73 & 0.706 & 85.45 & 15.15 & 97.86 \\
\hline & mTOR_VEGFR2_PDGFRB_ & & & & & & & \\
\hline & c-KIT_EGFR_FGFR1 & 6 & 55.56 & 82.94 & 0.730 & 81.82 & 12.20 & 97.77 \\
\hline & mTOR_VEGFR2_PDGFRB_c-KIT_ & & & & & & & \\
\hline & c-KIT_EGFR_FGFR1 & 7 & 88.89 & 58.29 & 0.747 & 59.55 & 8.33 & 99.19 \\
\hline
\end{tabular}

AUC, area under the curve; PPV, positive predictive value; NPV, negative predictive value; $m T O R$, mammalian target of rapamycin; VEGFR2, vascular endothelial growth factor receptor 2; $c$-KIT, KIT proto-oncogene receptor tyrosine kinase; $c$ - $R A F$, Raf-1 proto-oncogene serine/threonine kinase; FGFR1, fibroblast growth factor receptor 1; EGFR, epidermal growth factor receptor; $P D G F R B$, platelet-derived growth factor receptor beta. 


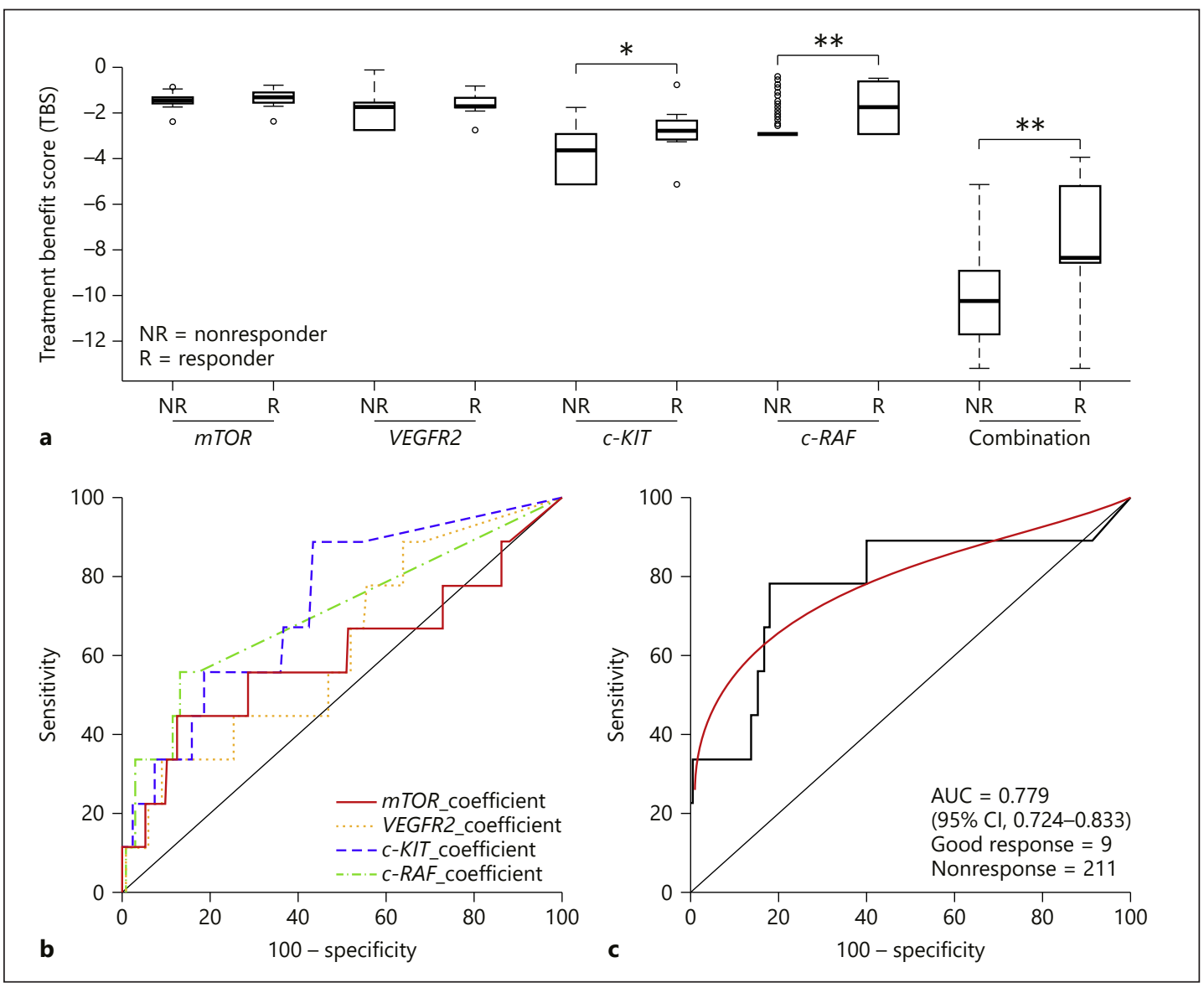

Fig. 4. Treatment benefit score (TBS) of seven actionable genes in responders versus nonresponders. a Relative TBS of seven actionable genes compared across 9 responders and 211 nonresponders. Scores were calculated as individual values for $m T O R, V E G F R 2, c-K I T$, and $c-R A F$, as well as paired values from $m T O R$ with VEGFR2 and $c$-KIT with $c$-RAF. The thick line in each box is the median. Circles extend to the most extreme data point, no more than 1.5 times the interquartile range. ${ }^{*} p<0.05,{ }^{* *} p<0.01$. b, c Receiver operating characteristic curve analysis to predict the response to sorafenib (TBS) per gene (b) and per pair (c). c $m$ TOR_VEGFR2_c-KIT_c-RAF.

Table 4. Logistic regression analysis of tumor response to sorafenib and variables

\begin{tabular}{llll}
\hline Variables & Odds ratio & $95 \%$ CI & $p$ value \\
\hline mTOR_VEGFR2_c-KIT_c-RAF (low vs. high) & 15.93 & $3.18-79.73$ & 0.002 \\
Age (<55 vs. $\geq 55$ years) & 1.44 & $0.35-5.93$ & 0.611 \\
Gender (male vs. female) & 1.83 & $0.44-7.62$ & 0.404 \\
HBV (absence vs. presence) & Inf. & $0.00-$ Inf. & 0.993 \\
HCV (absence vs. presence) & 0.00 & $0.00-$ Inf. & 0.994 \\
TNM stage (II-III vs. IV) & 0.82 & $0.16-4.11$ & 0.812 \\
BCLC stage (AB vs. CD) & 0.50 & $0.10-2.52$ & 0.399 \\
AFP level (<100 vs. $\geq 100 \mathrm{ng} / \mathrm{mL})$ & 0.52 & $0.12-2.21$ & 0.373 \\
\hline
\end{tabular}

CI, confidence interval; $m T O R$, mammalian target of rapamycin; VEGFR2, vascular endothelial growth factor receptor 2; $c$-KIT, KIT proto-oncogene receptor tyrosine kinase; $c-R A F$, Raf-1 proto-oncogene serine/ threonine kinase; HBV, hepatitis B virus; HCV, hepatitis C virus; TNM, Tumor, Node, and Metastasis classification; AJCC, American Joint Committee on Cancer; BCLC, Barcelona Clinic Liver Cancer; AFP, $\alpha$-fetoprotein. 
Table 5. Response rates based on gene classifier

\begin{tabular}{lllll}
\hline Classifier & Criterion & Responders & Nonresponders & Response rate, \% \\
\hline mTOR_VEGFR2_c-KIT_c-RAF & $>-8.6237$ & 7 & 38 & 15.6 \\
& $\leq-8.6237$ & 2 & 173 & 1.16 \\
\hline
\end{tabular}

mTOR, mammalian target of rapamycin; VEGFR2, vascular endothelial growth factor receptor 2; c-KIT, KIT proto-oncogene receptor tyrosine kinase; $c-R A F$, Raf-1 proto-oncogene serine/threonine kinase.

Improving the Predictability of TBS-Based Classifiers

Thus far, our findings have indicated that TBS can distinguish HCC patients with a complete response or partial response from those with stable disease or progressive disease, indicating that TBS-based patient selection should improve sorafenib's efficacy compared with the lack of any classification. When we examined 45 HCC patients with TBS >-8.6237 for $m T O R, V E G F R 2, c-K I T$, and $c$-RAF combinations, we found 7 responders (response rate $15.6 \%$; Table 5). The correlation between TBS and tumor responses to sorafenib was significant ( $p<$ $0.001)$.

\section{Discussion}

In this study, we found that TBS based on actionable gene expression successfully predict HCC tumor response to sorafenib. When the TBS was used for patient selection, the rate of response to sorafenib increased from 4.1 to $15.6 \%$. Our findings suggest that patient stratification based on actionable molecular subtyping is a useful therapeutic strategy for improving sorafenib's effectiveness in HCC, supporting our previous research [13].

An increased expression of actionable molecules (PDGFRB and $c$-KIT) in tumor cell lines may elevate tumor sensitivity to molecular targeted drugs. Based on the previous study, we investigated the mRNA expression of five actionable genes (VEGFR2, PDGFRB, EGFR, $m T O R$, and FGFR1) and two additional genes (c-KIT and $c$-RAF) in HCC tumors and surrounding noncancerous tissues (online suppl. Fig. 1, 2; for all online suppl. material, see www.karger.com/doi/10.1159/000504548). Then we conducted a seven-actionable-genebased study. Here, we observed a significantly higher mean expression of three actionable genes (PDGFRB, $c$-KIT, and $c-R A F)$ in responders than in nonresponders. This outcome is consistent with the fact that these three genes are direct sorafenib targets and associated with an enhanced response to sorafenib treatment [15]. However, mTOR and EGFR confer resistance to sorafenib through Akt activation [16,17], and, indeed, their expression did not differ significantly between responders and nonresponders, although the mean values were slightly higher in the responders (Fig. 3). Overall, our results suggest that mRNA levels of individual actionable genes are insufficient for predicting therapeutic response to sorafenib.

Although individual actionable genes were not useful biomarkers, our results demonstrated that the TBS of gene combinations were significantly higher in responders (Fig. 4a), with $m$ TOR/VEGFR2 and $c$-KIT/c-RAF achieving $77.8 \%$ sensitivity and $82.0 \%$ specificity (Fig. 4c; Table 3). These data are in line with the observation that multiple biomarkers usually outperform single biomarkers in diagnosis.

Furthermore, we demonstrated that adding VEGFR2, c-KIT, and $c-R A F$ to the $m T O R$ classifier could maximize predictions of response to sorafenib and thus improve treatment response rates. However, although the TBS raised the rates of response to sorafenib treatment from 4.1 to $15.6 \%$, a rate of $15.6 \%$ is still low in absolute terms. We believe that this low 
overall response rate is largely due to the small number of responders and heterogeneity in HCC patients (in contrast to the uniformity of HCC cell lines). Nevertheless, TBS based on actionable gene combinations exhibited a promising level of diagnostic performance in predicting response to sorafenib.

Given that sorafenib is the standard therapy for advanced HCC, excluding potential nonresponders might be the best approach to maximizing the drug's benefits. We therefore recommend against sorafenib treatment for patients below our suggested threshold values from our $m T O R / V E G F R 2$ and $c-K I T / c-R A F$ classifiers.

In conclusion, we have demonstrated the potential of actionable gene expression in predicting clinical responses to sorafenib treatment in HCC. Future research should aim to replicate these results using a larger gene pool. Nonetheless, our findings contribute to efforts aimed at improving diagnostic approaches that personalize sorafenib treatment of HCC.

\section{Acknowledgements}

The research was supported in part by the Korea Cancer Biomarker Consortium.

\section{Statement of Ethics}

The protocol was approved by the Institutional Review Boards of Ajou University Medical Center, Asan Medical Center, Gachon University Gil Medical Center, Korea University Medical Center, and Catholic University Medical Center. All patients provided written informed consent. The Declaration of Helsinki and Good Clinical Practice guidelines were followed.

\section{Disclosure Statement}

The authors have no conflicts of interest to declare.

\section{Funding Sources}

This work was supported by a National Research Foundation of Korea (NRF) grant funded by the Korean government (MEST) (Grant No. NRF-2013R1A1A4A01009053) and the Gil Medical Center, Gachon University College of Medicine (Grant No. CBS2013-10), South Korea.

\section{References}

1 Shin JW, Chung YH. Molecular targeted therapy for hepatocellular carcinoma: current and future. World J Gastroenterol. 2013 0ct;19(37):6144-55.

2 Ocana A, Pandiella A, Siu LL, Tannock IF. Preclinical development of molecular-targeted agents for cancer. Nat Rev Clin Oncol. 2010 Dec;8(4):200-9.

3 Sokolenko AP, Imyanitov EN. Molecular diagnostics in clinical oncology. Front Mol Biosci. 2018 Aug; 5:76.

4 Lynch TJ, Bell DW, Sordella R, Gurubhagavatula S, Okimoto RA, Brannigan BW, et al. Activating mutations in the epidermal growth factor receptor underlying responsiveness of non-small-cell lung cancer to gefitinib. $\mathrm{N}$ Engl J Med. 2004 May;350(21):2129-39.

5 Tsao H, Chin L, Garraway LA, Fisher DE. Melanoma: from mutations to medicine. Genes Dev. 2012 Jun;26(11): 1131-55.

6 Llovet JM, Ricci S, Mazzaferro V, Hilgard P, Gane E, Blanc JF, et al.; SHARP Investigators Study Group. Sorafenib in advanced hepatocellular carcinoma. N Engl J Med. 2008 Jul;359(4):378-90.

7 Cheng AL, Kang YK, Chen Z, Tsao CJ, Qin S, Kim JS, et al. Efficacy and safety of sorafenib in patients in the AsiaPacific region with advanced hepatocellular carcinoma: a phase III randomised, double-blind, placebocontrolled trial. Lancet Oncol. 2009 Jan;10(1):25-34. 
8 Kudo M, Finn RS, Qin S, Han KH, Ikeda K, Piscaglia F, et al. Lenvatinib versus sorafenib in first-line treatment of patients with unresectable hepatocellular carcinoma: a randomised phase 3 non-inferiority trial. Lancet. 2018 Mar;391(10126):1163-73.

9 Forner A, Reig M, Bruix J. Hepatocellular carcinoma. Lancet. 2018 Mar;391(10127):1301-14.

10 Marisi G, Cucchetti A, Ulivi P, Canale M, Cabibbo G, Solaini L, et al. Ten years of sorafenib in hepatocellular carcinoma: are there any predictive and/or prognostic markers? World J Gastroenterol. 2018 Sep;24(36): 4152-63.

11 Horwitz E, Stein I, Andreozzi M, Nemeth J, Shoham A, Pappo O, et al. Human and mouse VEGFA-amplified hepatocellular carcinomas are highly sensitive to sorafenib treatment. Cancer Discov. 2014 Jun;4(6):730-43.

12 Arao T, Ueshima K, Matsumoto K, Nagai T, Kimura H, Hagiwara S, et al. FGF3/FGF4 amplification and multiple lung metastases in responders to sorafenib in hepatocellular carcinoma. Hepatology. 2013 Apr;57(4):140715.

13 Kwon JH, Lee N, Park JY, Yu YS, Kim JP, Shin JH, et al. Actionable gene expression-based patient stratification for molecular targeted therapy in hepatocellular carcinoma. PLoS One. 2013 Jun;8(6):e64260.

14 Lencioni R, Llovet JM. Modified RECIST (mRECIST) assessment for hepatocellular carcinoma. Semin Liver Dis. 2010 Feb;30(1):52-60.

15 Wilhelm SM, Adnane L, Newell P, Villanueva A, Llovet JM, Lynch M. Preclinical overview of sorafenib, a multikinase inhibitor that targets both Raf and VEGF and PDGF receptor tyrosine kinase signaling. Mol Cancer Ther. 2008 Oct; $7(10): 3129-40$.

16 Masuda M, Chen WY, Miyanaga A, Nakamura Y, Kawasaki K, Sakuma T, et al. Alternative mammalian target of rapamycin (mTOR) signal activation in sorafenib-resistant hepatocellular carcinoma cells revealed by arraybased pathway profiling. Mol Cell Proteomics. 2014 Jun;13(6):1429-38.

17 Ezzoukhry Z, Louandre C, Trécherel E, Godin C, Chauffert B, Dupont S, et al. EGFR activation is a potential determinant of primary resistance of hepatocellular carcinoma cells to sorafenib. Int J Cancer. 2012 Dec; 131(12):2961-9. 